\title{
Deletion of Lactate Dehydrogenase in Klebsiella Pneumoniae to Enhance 1,3- Propanediol Production
}

\author{
Guangbin Ye $\mathrm{Y}^{1, \mathrm{a}}$, Shanshan Sun ${ }^{1, \mathrm{~b}}$, Liting Deng ${ }^{1, \mathrm{c}}$, \\ ${ }^{1}$ Key Laboratory of Microbiology, College of Life \\ Science, \\ Heilongjiang University, \\ Harbin, 150080, China \\ a 854319559@qq.com, b907777522@qq.com, \\ c252817006@qq.com,
}

\author{
Jingping $\mathrm{Ge}^{*^{1,2, \mathrm{~d}} \text {, Wenxiang Ping, }}{ }^{1,2, \mathrm{e}}$ \\ ${ }^{2}$ Engineering Research Center of Agricultural \\ Microbiology Technology, Ministry of Education, \\ Harbin 150080, China \\ ewenxiangping@aliyun.com, \\ *Corresponding author: gejingping@126.com
}

\begin{abstract}
Propanediol (1,3-PD) is an important chemical intermediate in manufacturing polyethers, polyurethanes and polyesters. Production of 1,3-PD by microbial fermentation has attracted increasingly attention of researchers. In this study, deletion mutant of K.pneumoniae HD-1 constructed by knocking out lactate dehydrogenase (LDH) gene in our previous work was cultivated in fermentation medium utilizing glucose as carton source and original strain K.pneumoniae were simultaneously used as the control strain under absolutely the same conditions and their capability of 1,3-PD production were compared. The results showed that an increase of $19.98 \%, 20 \%$, and $20.19 \%$ of 1,3-PD titer, productivity, and yield, respectively were obtained from K.pneumoniae HD-1. Obviously it can be inferred that the ability in the production of 1,3-PD of the K.pneumoniae HD-1was better than that of the K.pneumoniae, therefore it could be a promising strain for 1,3-PD production in the further study of industrialized production.
\end{abstract}

Keywords-K.pneumoniae; lactate dehydrogenase; 1,3propanediol; batch fermentation.

\section{INTRODUCTION}

1,3-Propanediol (1,3-PD) is an important chemical intermediate in manufacturing polyethers, polyurethanes and polyesters. It is widely applied in food, cosmetic and pharmaceutical industries ${ }^{[1]}$. Besides, 1,3-PD can be used as intermediate to synthesize biocides and heterocyclic compounds and it is extensively used in the manufacture of polytrimethylene terephthalate (PTT) which demonstrates excellent potential of stretching and stretchrecovering in producing fiber for textiles and carpets that has been known as "new nylon" currently ${ }^{[2-3]}$. In addition, compared with the commercial polyester from market, synthesis of polyester from 1,3-PD has a higher efficiency to reduce dyeing temperature, the higher the UV resistance and better fastness ${ }^{[4]}$.

Many microorganisms can be served as 1,3-PD producers when anaerobically cultivated in fermentation medium, while K.pneumoniae shows excellent ability to produce 1,3-PD with higher yield and productivity ${ }^{[5]}$. There are primarily two branch pathways that are the reductive branch and the oxidative branch involved in glycerol metabolic pathway in K.pneumoniae which has been comprehensive studied ${ }^{[6-7]}$. In the reductive branch, 3-hydroxypropanal (3-HPA) is firstly synthesized by the conversion of glycerol at the catalysis of glycerol dehydratase which is then formed to 1,3-PD by 1,3propanediol oxidoreductase ${ }^{[8]}$. However, a series of byproducts such as 2,3-butanediol (2,3-BD), acetate, lactate are produced due to the existence of oxidative branch, which lead to transforming of the carbon flux and the decreasing of 1,3-PD yield. It is mainly the accumulation of lactate in the stable period of cell growth that decreased the 1,3-PD productivity and thus will be costly in downstream purification process ${ }^{[9]}$. It is of great significance to prevent the synthesis of lactate in 1,3-PD production $^{[10]}$.

In this study, deletion mutant of K.pneumoniae HD-1 which was constructed by knocking out lactate dehydrogenase gene applying integrational mutagenesis to K.pneumoniae HD-1 in previously experiment in our laboratory and original strain K.pneumoniae were simultaneously used as strains in the fermentation medium utilizing glucose as carton source under absolutely the same conditions, and 1,3-PD production capability were compared to verify whether the construction of K.pneumoniae HD-1 was successful and whether the gene expression of the deletion mutant was stable. The one with better ability to produce 1,3-PD will be chosen for further study on improving the yield of 1,3PD.

\section{MATERIALS AND METHODS}

\section{A. Microorganisms and medium}

K.pneumoniae and K.pneumoniae HD-1 were used in the work. The seed cells were prepared in 250 ml flasks containing $100 \mathrm{ml}$ pre-culture medium which contained (g/L): $6.0\left(\mathrm{NH}_{4}\right)_{2} \mathrm{HPO}_{4}, 0.51$ EDTA, $1.8 \mathrm{KCl}, 0.29$ sodium citrate, $0.6 \mathrm{MgSO}_{4} \cdot 7 \mathrm{H}_{2} \mathrm{O}, 0.21$ citric acid, 1.5 yeast extract, 30 glucose, $1 \mathrm{ml}$ microelement solution, $1 \mathrm{ml}$ $\mathrm{Fe}^{3+}$ solution. The flasks were incubated at $37^{\circ} \mathrm{C}$ for $24 \mathrm{~h}$ and inoculated into the fermentation medium at $5 \%(\mathrm{v} / \mathrm{v})$, the fermentation medium containing(g/L):6.6 $\left(\mathrm{NH}_{4}\right)_{2} \mathrm{SO}_{4}$, $0.69 \mathrm{~K}_{2} \mathrm{HPO}_{4} \cdot 3 \mathrm{H}_{2} \mathrm{O}, 1.36 \mathrm{KH}_{2} \mathrm{PO}_{4}, 0.26 \mathrm{MgSO}_{4} \cdot 7 \mathrm{H}_{2} \mathrm{O}$, 0.43 citric acid, 1.0 yeast extract, $1 \mathrm{ml}$ microelement solution, $1 \mathrm{ml} \mathrm{Fe}^{3+}$ solution, 60 glucose. 


\section{B. Batch fermentation experiment}

The batch fermentation process was performed in 500 $\mathrm{ml}$ flasks containing $150 \mathrm{ml}$ fermentation medium with initial $\mathrm{pH} 6.8$ and cultured at $37^{\circ} \mathrm{C}$ with a shaking speed of $150 \mathrm{rpm}$ for $108 \mathrm{~h}$. Samples were harvested every 12 hours and the $\mathrm{pH}$ was maintained using sterile $\mathrm{CaCO}_{3}$ powder depending on the experiment.

\section{Analytical methods}

Cell growth was described by an optical density (OD) calibration curve determined at $650 \mathrm{~nm}$. The concentrations of glucose, 1,3-PD, 2,3-BD, ethanol, acetate, succinate, and lactate obtained from the batch fermentation process were determined by a highperformance liquid chromatography (HPLC) System (Shimadzu Corp. Kyoto, Japan). Aminex HPX-87H column (Bio-Rad, Hercules, CA, USA) was used with $0.005 \mathrm{M} \mathrm{H}_{2} \mathrm{SO}_{4}$ as elution solvent at a flow rate of 0.8 $\mathrm{ml} / \mathrm{min}$, keeping column oven temperature at $65^{\circ} \mathrm{C}$ with RI detector.

\section{Measurement of relative enzyme activities}

For enzyme activities determination, the precipitate from $6 \mathrm{~mL}$ fermentation broth that was centrifuged at $5,000 \mathrm{rpm}$ for $10 \mathrm{~min}$ at $4^{\circ} \mathrm{C}$ was washed twice with potassium phosphate buffer $(100 \mathrm{mM}, \mathrm{pH} 7.4)$ and then was resuspended with the buffer. The supernatant from broken Cells which was performed by ultrasonic treatment in ice water bath after centrifugation $(10,000$ $\mathrm{r} / \mathrm{m}, 10 \mathrm{~min}$ at $4^{\circ} \mathrm{C}$ ) was used as crude enzymes liquor for the measurement of enzymes activities. The total protein concentration was determined by the Bradford method with bovine serum albumin as the standard. One unit of enzyme is defined as the amount of enzyme required to produce/consume $1 \mathrm{mmol}$ of product/substrate per minute $^{[11]}$.

\section{RESULTS AND DISCUSSION}

\section{A. LDH activity assays}

The LDH activities of the parent strain and the mutant were determined. As shown in Table.1, the LDH activities were 0.232 and 0.128 of the mutant and the parent strain. The enzymatic analysis further proved that ldhA had been knocked out partially by integrational mutagenesis, resulting in the decrease of the LDH enzyme activity.

TABLE 1. LDH ACTIVITIES OF K.PNEUMONIAE AND K.PNEUMONIAE HD-

\begin{tabular}{ccc}
\hline & \multicolumn{2}{c}{ strains } \\
\cline { 2 - 3 } & K.pneumoniae & K.pneumoniae HD-1 \\
\hline $\begin{array}{c}\text { LDH activity(U/mg } \\
\text { protein) }\end{array}$ & $0.232 \pm 0.02$ & $0.128 \pm 0.03$ \\
\hline
\end{tabular}

B. The production of $1,3-P D$ in batch fermentation.

Growth curve of strains and $\mathrm{pH}$ value in the fermentation process were shown in Figure.1.

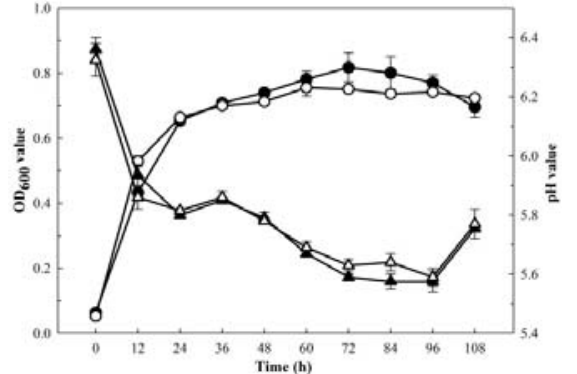

Figure 1. Batch fermentation of K.pneumoniae and K.pneumoniae HD-1. Closed circle, cell growth of K.pneumoniae; open circle, cell growth of K.pneumoniae HD-1; closed Triangle-up, $\mathrm{pH}$ value of K.pneumoniae; open Triangle-up, $\mathrm{pH}$ value of K.pneumoniae HD-1. The values were the mean of two independent samples.

The results of typical end-products in micro-aerobic batch fermentations were shown in Fig. 2 and the average values with $95 \%$ confidence intervals were listed in Table.2.
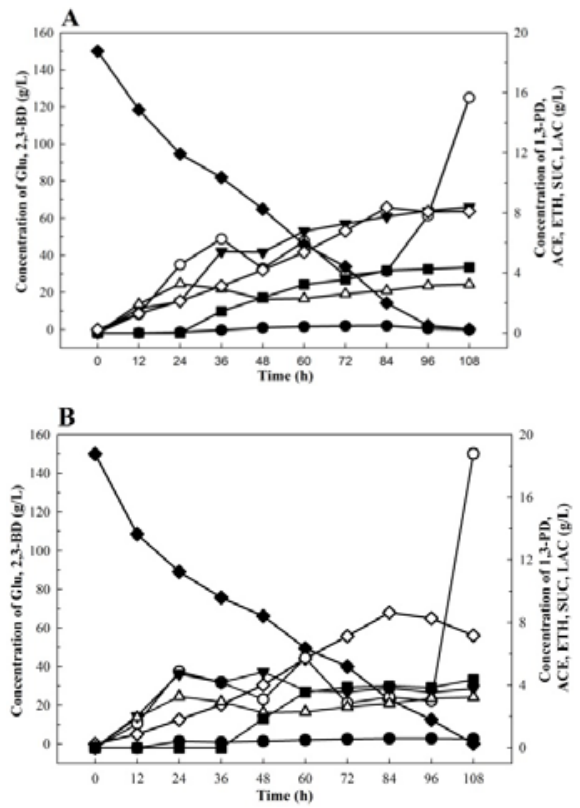

Figure 2. Time courses of K.pneumoniae (A) and K.pneumoniae HD-1 (B) batch fermentation under aerobic condition. Closed circle, succinate; open circle, 1,3-PD; square, acetate; closed diamonds, glucose; open diamonds, 2,3-BD; closed triangles down, lactate; open triangles up, ethanol. 
TABLE 2. COMPARISON OF BATCH FERMENTATION BY K.PNEUMONIAE AND K.PNEUMONIAE HD-1

\begin{tabular}{ccc}
\hline & \multicolumn{2}{c}{ Strains } \\
\cline { 2 - 3 } & K.pneumoniae & K.pneumoniae \\
& & $150 \pm 0.001$ \\
\hline Glucose consumed (g/L) & $150 \pm 0.001$ & $18.79 \pm 0.026$ \\
1,3-PD titer (g/L) & $15.66 \pm 0.015$ & $68.03 \pm 0.009$ \\
2,3-BD titer (g/L) & $65.75 \pm 0.031$ & $4.84 \pm 0.001$ \\
Lactate titer (g/L) & $8.39 \pm 0.001$ & $0.591 \pm 0.005$ \\
succinate titer (g/L) & $0.486 \pm 0.002$ & $0.125 \pm 0.001$ \\
1,3-PD yield (g/g) & $0.104 \pm 0.002$ & $0.174 \pm 0.03$ \\
1,3-PD & $0.145 \pm 0.02$ & \\
productivity(g/L·h) & & \\
\hline
\end{tabular}

Theoretically the formation of lactate can be prevented by inactivating lactate dehydrogenase. The K.pneumoniae HD-1 was constructed by disrupting ldhA gene that encodes lactate dehydrogenase, which showed markedly lower lactate production $(4.84 \mathrm{~g} / \mathrm{L})$ compared to the original strain $(8.39 \mathrm{~g} / \mathrm{L})$. Concomitantly, the finally 1,3PD titer and yield increased from 15.66 to $18.79 \mathrm{~g} / \mathrm{L}$ and 0.104 to $0.125 \mathrm{~g} / \mathrm{g}$ respectively. The increasing of other two important products that were 2,3-BD and succinate were $3.47 \%$ and $21.6 \%$. In order to evaluate and compare the performance of 1,3-PD fermentation between K.pneumoniae and K.pneumoniae HD-1, the total glucose uptake concentration and 1,3-PD productivity were determined at the time the glucose was completely depleted in the fermentation broth (initially $150 \mathrm{~g} / \mathrm{L}$ ). And the total glucose uptake rate of the mutant was lower than the wild-type strain which led to the biomass of the mutant (Fig.1) and the 1,3-PD productivity was slightly higher from 0.145 to $0.174 \mathrm{~g} / \mathrm{L} \cdot \mathrm{h}$. Furthermore, the final concentrations of succinate increased to $0.105 \mathrm{~g} / \mathrm{L}$ of the mutant while other by-products such as ethanol. and acetate were nearly similar to the wild-type strain.

Lactate was the main by-product in 1,3-PD production by K.pneumoniae, which competed for NADH against 1,3-PD pathway and certainly reduced the yield of 1,3-PD from glucose. In this study, K.pneumoniae HD-1 lacking fermentative lactate dehydrogenase was constructed and isolated to assess the physiological importance of lactate production and thus making the production of 1,3-PD more efficient. Synthesis of 1,3-PD plays significant role in regulating the balance of intracellular reducing equivalent by consuming $\mathrm{NADH}$ generated from the oxidation pathway (Zeng et al, 1993) ${ }^{[12]}$. Furthermore, considerable quantity of pyruvate which is an important metabolize intermediate flowed to 2,3-BD pathway as the lactate synthesis pathway was cut off and part of the excessive NADH could be utilized. Thereby, 2,3-BD which is also known as a fuel additive with high valueadded became the main by-product in K.pneumoniae HD1 fermentation ${ }^{[13]}$.

\section{CONCLUSIONS}

K.pneumoniae HD-1 lacking fermentative lactate dehydrogenase (LDH) exhibited higher 1,3-PD productivity and less lactate accumulation. In addition, 2,3-BD and succinate has also slightly increased. The results showed that the ability in 1,3-PD production of genetic engineering strains was better than wild-type strain, which simultaneously was more suitable for further research of higher production of 1,3-PD.

\section{ACKNOWLEDGEMENTS}

The research was supported by The National Natural Science Foundation of China(Grant No.31270534), The National Natural Science Foundation of China (Grant No. 31270143), The National Natural Science Foundation of China (Grant No.31470537), and the National Science Foundation for Distinguished Young Scholars of China(31300355).

\section{REFERENCES}

[1] R.K. Saxena, Pinki Anand, et al. Microbial production of 1,3propanediol: Recent developments and emerging opportunities[J]. Biotechnology Advances 27 (2009):895-913.

[2] Jalasutram V, Jetty A, Anupoju G.R. Fermentative conversion of raw glycerol into 1,3-propanediol by isolated Klebsiella pneumoniae 141B stain: optimization of culture variables[J]. Afr J Biotechnol 2011, 10:493-502

[3] Hao J, Lin RH, Zheng ZM, et al. Isolation and characterization of microorganisms able to produce 1,3-propanediol under aerobic conditions[J]. World J Microbiol Biotechnol 2008a, 24:1731-1740.

[4] Hao J, Liu HJ, Liu DH. Novel route of reactive extraction to recover 1,3-propanediol from a dilute aqueous solution[J]. Ind Eng Chem Res 2005, 44:4380-4385.

[5] C.S. Leea, M.K. Aroua, et al. A review: Conversion of bioglycerol into 1,3-propanediol via biological and chemical method[J]. Renewable and Sustainable Energy Reviews 42 (2015) 963-972.

[6] Zeng AP, Menzel K, Deckwer WD. Kinetic, dynamic, and pathway studies of glycerol metabolism by Klebsiella pneumoniae in anaerobic continuous culture: II. Analysis of metabolic rates and pathways under oscillation and steady-state conditions[J]. Biotechnol Bioeng, 1996, 52:561-571.

[7] Qingrui Zhang, Zhilong Xiu. Metabolic Pathway Analysis of Glycerol Metabolism in Klebsiella pneumonia Incorporating Oxygen Regulatory System[J]. Biotechnol Prog. 2009, 25(1):103115.

[8] Zheng ZM, Cheng KK, Liu DH, et al. Effect of culture conditions on 3-hydroxypropionaldehyde detoxification in 1,3-propanediol fermentation by Klebsiella pneumonia[J]. Biochem Eng J, 2008, 39:305-310.

[9] Ni-Ni Guo, Zong-Ming Zheng, Yu-Lin Mai, et al. Consequences of cps mutation of Klebsiella pneumonia on 1,3-propanediol fermentation[J]. Appl Microbiol Biotechnol (2010) 86:701-707.

[10] Jiang G R, Nikolova S, Clark D P. Regulation of the IdhA gene, encoding the fermentative lactate dehydrogenase of Escherichia coli[J]. Microbiology 2001, 147:2437-2446.

[11] Yun-Zhen Xu, Ni-Ni Guo, Zong-Ming Zheng, et al. Metabolism in 1,3-Propanediol Fed-Batch Fermentation by a D-Lactate Deficient Mutant of Klebsiella pneumonia[J]. Biotechnology and Bioengineering, 2009, 104(5): 965-971.

[12] Zeng AP, Biebl H, Schlieker H, Deckwer WD. Pathway analysis of glycerol fermentation by Klebsiella pneumoniae: Regulation of reducing equivalent balance and product formation[J]. Enzyme Microb Technol, 1993, 15:770-779.

[13] Syu U M J. Biological production of 2,3-Butanediol[J]. Applied Microbiol and Biotechnol, 2001, 55(1): 10-18. 